\title{
Neutrophil to lymphocyte ratio in the pre-treatment phase of final-line chemotherapy predicts the outcome of patients with recurrent ovarian cancer
}

\author{
KEIICHIRO NAKAMURA ${ }^{1}$, TAKESHI NAGASAKA ${ }^{2}$, TAKESHI NISHIDA ${ }^{1}$, TOMOKO HARUMA ${ }^{1}$, \\ CHIKAKO OGAWA ${ }^{1}$, TOMOYUKI KUSUMOTO ${ }^{1}$, NORIKO SEKI ${ }^{1}$ and YUJI HIRAMATSU ${ }^{1}$ \\ ${ }^{1}$ Department of Obstetrics and Gynecology, ${ }^{2}$ Department of Gastroenterological Surgery, \\ Okayama University Graduate School of Medicine, Dentistry and Pharmaceutical Sciences, \\ Okayama, Okayama 700-8558, Japan
}

Received January 9, 2015; Accepted October 19, 2015

DOI: $10.3892 / 01.2016 .4513$

\begin{abstract}
Inflammation and tumor immunology are associated with prognosis in a variety of cancers. The aim of the present retrospective study was to identify associations between the neutrophil to lymphocyte ratio (NLR), platelet to lymphocyte ratio (PLR), cancer antigen 125 (CA125) concentrations, tumor response, performance status (PS) and survival of patients that developed recurrent ovarian cancer subsequent to receiving chemotherapy. The NLR and PLR measured prior to fourth-line chemotherapy were significantly increased compared with those measured prior to second-line chemotherapy ( $\mathrm{P}=0.029$ and 0.049 , respectively). By using receiver operating characteristic curves, the cut-off values were determined for the NLR, PLR and CA125 levels that were measured during the pre-treatment phase, which predicted the outcomes. According to univariate analyses, pre-treatment NLR $>3.91$, PLR $>299.0$ and PS 2 were each significantly associated with poor outcomes $(\mathrm{P}=0.001$, 0.005 and 0.021 , respectively). According to multivariate analyses, only pre-treatment NLR was associated with poor outcome $(\mathrm{P}=0.035)$. The present findings indicate that pre-treatment NLR is an important predictor of prognosis in patients with ovarian cancer that experience recurrence following chemotherapy.
\end{abstract}

Correspondence to: Dr Keiichiro Nakamura, Department of Obstetrics and Gynecology, Okayama University Graduate School of Medicine, Dentistry and Pharmaceutical Sciences, 2-5-1 Shikata-cho, Okayama, Okayama 700-8558, Japan.

E-mail: k-nakamu@cc.okayama-u.ac.jp

Key words: recurrent ovarian cancer, chemotherapy, neutrophil to lymphocyte ratio, predictor of survival

\section{Introduction}

Ovarian cancer patients demonstrate poor survival; the five-year survival rate for patients with advanced stage [FIGO stage III-IV (1)] disease is just $30.6 \%(2,3)$. Despite a high initial response rate to primary treatments, $75 \%$ of patients eventually relapse and succumb to the disease $(2,4)$. Responses to second-line chemotherapy for recurrence occur in $\geq 60 \%$ of platinum-sensitive patients, with $\sim 25 \%$ of these patients achieving complete responses $(5,6)$. Although there is much published data on the survival outcomes of various third-line chemotherapy options, limited information is available specifically regarding the response to chemotherapy. Generally, chemotherapy is considered the best initial treatment option for patients with incurable cancer (7-9). The challenge for the physician is to continuously balance the pros and cons in each individual patient, so as to optimize tumor response while minimizing adverse effects. Decisions about chemotherapy should consider various clinical factors, including the performance status (PS) of the patient and the likely tumor response and patient survival time. In particular, the prediction of the survival time is important. Evidence of deterioration and adverse effects should be monitored closely to avoid overtreatment (8).

In addition to clinical predictors of the survival duration, such as PS, possible prognostic biomarkers associated with the systemic inflammatory processes have been examined in various types of cancer, as inflammation in tumors has previously been identified to enable various cancer characteristics and to be important in determining the prognosis (10). Changes in the proportions of the subtypes of white blood cells have been the most frequently evaluated of these biomarkers, as they are easily assessable and economically feasible potential markers of cancer prognosis $(11,12)$. The neutrophil to lymphocyte ratio (NLR) and the platelet to lymphocyte ratio (PLR) have attracted the interest of studies as systemic inflammatory markers of prognosis $(13,14)$. Several studies have reported that peripheral blood NLR and PLR measured during the pre-operative or pre-treatment phase are independent predictors of poor prognosis in various 
cancers, including pancreatic, breast, colon, lung and gastric cancer (15-19). However, associations between the NLR and PLR and the tumor responses and survival of patients receiving chemotherapy have not been examined.

Therefore, in the present study, the association between the NLR and PLR and the PS, tumor response and survival time were investigated in patients with recurrent epithelial ovarian cancer subsequent to the administration of chemotherapy, in which the response to chemotherapy had been monitored by serial cancer antigen 125 (CA125) concentrations (20).

\section{Patients and methods}

Patients. The present study was a retrospective analysis of 30 ovarian cancer patients with recurrence that had been treated in the Department of Obstetrics and Gynecology of Okayama University Hospital (Okayama, Okayama, Japan) between April 2005 and April 2013. The primary treatment received by these patients was complete cytoreductive surgery and adjuvant and/or neo-adjuvant chemotherapy with conventional taxotere and cyclophosphamide (TC) $\left[180 \mathrm{mg} / \mathrm{m}^{2}\right.$ paclitaxel infused over $3 \mathrm{~h}$; carboplatin, dosage calculated for an area under the curve (AUC) of 5 infused over $1 \mathrm{~h}$; Bristol-Myers Squibb, New York, NY, USA]. Following primary treatment, the patients underwent follow-up examinations every 1-2 months for the first 6 months, every 3 months for the next 2 years, and every 6 months thereafter. The present protocol was approved by the Institutional Review Board of Okayama University Hospital. Informed consent was obtained from all patients.

Chemotherapy. The policy of the Department of Obstetrics and Gynecology of Okayama University Hospital required an Eastern Cooperative Oncology Group PS of $\leq 2$ (21) prior to initiating chemotherapy. In the present study, no patients showed clinical or radiographic evidence of relapse within 6 months subsequent to completing adjuvant and/or neo-adjuvant chemotherapy with conventional TC. Conventional TC was also administered as second-line chemotherapy to patients that developed evidence of clinical or radiographic relapse within the 6 months subsequent to completing adjuvant and/or neo-adjuvant chemotherapy. Chemotherapy for the treatment of recurrent disease was continued until complete response (CR) or progressive disease (PD) was identified. Patients with $\mathrm{PD}$ received regimens of chemotherapy that were different from the adjuvant and second-line combinations. The second line chemotherapy consisted of weekly TC (paclitaxel, $80 \mathrm{mg} / \mathrm{m}^{2}$; carboplatin, AUC of 2.0). Third, fourth and fifth-line chemotherapy was either single agent irinotecan (CPT-11; $70 \mathrm{mg} / \mathrm{m}^{2}$ weekly for 3 weeks followed by 1 week off; Yakult, Tokyo, Japan), pegylated liposomal doxorubicin (Doxil; $40 \mathrm{mg} / \mathrm{m}^{2}$ once every four weeks; Janssen, Tokyo, Japan), or gemcitabine $\left(700 \mathrm{mg} / \mathrm{m}^{2}\right.$ weekly for 3 weeks followed by 1 week off; Eli Lilly and Company, Indianapolis, IN, USA). The objective responses of the patients were principally evaluated using the Response Evaluation Criteria in Solid Tumors guidelines (version 1.1) (22). Patients with recurrence that occurred in a single site, and therefore underwent secondary surgical cytoreduction, and those in whom granulocyte colony stimulating factor was used were excluded from the present study.
Table I. Characteristics of patients with ovarian cancer.

\begin{tabular}{lc}
\hline Baseline characteristics & Patients, n (\%) \\
\hline Age at diagnosis, mean years (range) & $54.9(33-78)$ \\
Histology & \\
High grade serous adenocarcinoma & $30(100.0)$ \\
RFS time & \\
7-12 months & $23(76.7)$ \\
$13-24$ months & $5(16.6)$ \\
$>25$ months & $2(6.7)$ \\
\hline
\end{tabular}

RFS, reccurence-free survival; TC, paclitaxel + cisplatin.

NLR, PLR and CA125. All subjects underwent complete blood and differential white blood cell counts prior to commencing chemotherapy. The NLR was defined as the absolute neutrophil count divided by the absolute lymphocyte count, and the PLR was defined as the absolute platelet count divided by the lymphocyte count (Bayer HealthCare, Tarrytown, NY, USA). Serum CA125 concentrations were measured using an electrochemiluminescence immunoassay using Hitachi Modular Analytics E170 (Roche Diagnostics, Tokyo, Japan). Additionally, NLR, PLR and CA125 concentrations were measured prior to commencing treatment for recurrent ovarian cancer. Since objectively measurable criteria for assessing the response to chemotherapy were considered important, changes in the NLR and PLR values and CA125 concentrations were evaluated as single prognostic criteria and in combination with other clinical response criteria.

Statistical analysis. Statistical analyses were performed using the Mann-Whitney U-test for comparisons with controls and one-way analysis of variance followed by Fisher's protected least significant difference test for all pairwise comparisons. Receiver operating characteristic (ROC) curves were generated for pre-treatment NLR, PLR and CA125 concentrations prior to final line chemotherapy to determine the cut-off values for predicting survival for $>100$ days that yielded optimal sensitivity and specificity. The patients were then allocated to groups based on the cut-off values. Univariate and multivariate analyses using Cox's proportional hazards model were performed to determine the biomarkers that predicted survival subsequent to adjusting for the effects of known prognostic factors. Analyses were performed using SPSS software version 20.0 (IBM, Armonk, NY, USA). P<0.05 was considered to indicate a statistically significant difference.

\section{Results}

Patient characteristics. The histological types of ovarian cancer included in the present study and the duration of recurrence-free survival are listed in Table I. Following disease recurrence, 30 (100\%), 28 (93.3\%), 21 (70.0\%) and $10(33.3 \%)$ patients received second, third, fourth and fifth-line chemotherapy, respectively (Fig. 1A). The tumor responses of patients that received second to fifth-line chemotherapy are exhibited in Fig. 1B. The tumor response and disease control rates were 
Table II. Prognostic factors for mortality within 100 days of the failure of final-line chemotherapy.

\begin{tabular}{|c|c|c|c|c|c|c|}
\hline \multirow[b]{2}{*}{ Variable } & \multicolumn{3}{|c|}{ Univariate analysis } & \multicolumn{3}{|c|}{ Multivariate analysis } \\
\hline & Hazard ratio & $95 \% \mathrm{CI}$ & P-value & Hazard ratio & $95 \% \mathrm{CI}$ & P-value \\
\hline Years (>60 years) & 1.202 & $0.339-4.264$ & 0.776 & & & \\
\hline RFS ( $\leq 12$ months) & 3.339 & $0.423-26.376$ & 0.253 & & & \\
\hline Over fourth line chemotherapy & 0.905 & $0.234-3.509$ & 0.886 & & & \\
\hline Pre-treatment NLR (>3.91) & 31.667 & $3.887-257.963$ & $0.001^{*}$ & 14.128 & $1.207-165.400$ & $0.035^{*}$ \\
\hline Pre-treatment PLR (>299.0) & 19.173 & $2.396-153.423$ & $0.005^{*}$ & 4.628 & $0.432-49.567$ & 0.205 \\
\hline CA125 (>722.0) & 1.032 & $0.299-3.568$ & 0.960 & & & \\
\hline PS (2) & 4.362 & $1.248-15.240$ & $0.021^{*}$ & 0.974 & $0.258-3.667$ & 0.969 \\
\hline
\end{tabular}

Asterisks denote significance. RFS, reccurence free survival; NLR, neutrophil to lymphocyte ratio; PLR, platelet to lymhocyte ratio; PS, performance status; CI, confidence interval.
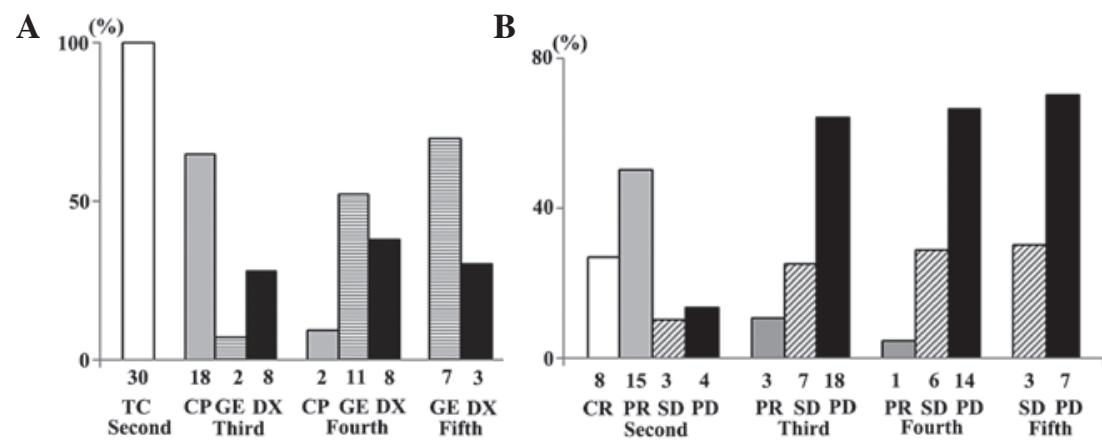

Figure 1. (A) Second to fifth-line chemotherapy regimens used to treat recurrent ovarian cancer. (B) Responses to second to fifth-line chemotherapy in patients with recurrent ovarian cancer. TC, paclitaxel + carboplatin; CP, irinotecan; DX, pegylated liposomal doxorubicin; GE, gemcitabine; CR, complete response; $\mathrm{PR}$, partial response; $\mathrm{SD}$, stable disease; $\mathrm{PD}$, progressive disease.

76.7 and $86.7 \%$, respectively, for second-line chemotherapy, 10.7 and $35.7 \%$, respectively, for third-line chemotherapy, 4.3 and $33.3 \%$, respectively, for fourth-line chemotherapy, and 0.0 and $30.0 \%$, respectively, for fifth-line chemotherapy. The median number of cycles of second, third, fourth and fifth-line chemotherapy received were 16 (range, 5-27), 7 (range, 2-27), 6 (range, 2-20), and 5 (range, 2-18), respectively.

Association between chemotherapy line and the NLR, PLR and CA125 concentration of patients. The pre-treatment NLR, PLR and CA125 concentrations were calculated for second to fifth-line chemotherapy. The mean pre-treatment NLR ( \pm 2 SD) for second, third, fourth and fifth-line chemotherapy was $2.24 \pm 0.74,2.64 \pm 1.56,3.12 \pm 1.72$ and $3.66 \pm 2.70$, respectively. The mean pre-treatment PLR ( $\pm 2 \mathrm{SD})$ for second, third, fourth and fifth-line chemotherapy was 188.6 \pm 87.5 , $246.8 \pm 152.2,284.2 \pm 210.8$ and $293.2 \pm 182.5$, respectively. The mean pre-treatment CA125 concentration ( $\pm 2 \mathrm{SD}$ ) for second, third, fourth and fifth-line chemotherapy was $238.1 \pm 385.6$, $597.0 \pm 808.0,1539.2 \pm 1568.6$, and $1919.0 \pm 2462.0 \mathrm{U} / \mathrm{ml}$, respectively. The pre-treatment NLR and PLR for fourth-line chemotherapy were significantly increased compared with those for second-line chemotherapy $(\mathrm{P}=0.029$ and 0.049 , respectively). The mean pre-treatment CA125 concentrations for third and fourth-line chemotherapy were significantly increased compared with the mean pre-treatment CA125 concentrations for second-line chemotherapy $(\mathrm{P}=0.039$ and 0.001 , respectively) (Fig. 2A).

Whether the pre-treatment NLR, PLR and CA125 concentrations for second to fifth-line chemotherapy were associated with the disease control rate (DCR), including CR, partial response (PR), and stable disease (SD) was assessed. For third-line chemotherapy, the mean pre-treatment NLR of patients with subsequent PD was increased compared with the mean pre-treatment NLR for patients that achieved CR, PR, and SD $(P=0.022)$. Similarly, the mean pre-treatment PLR for patients with subsequent PD was significantly increased compared with the mean pre-treatment PLR for patients that achieved CR, PR, and SD with fourth-line chemotherapy $(\mathrm{P}=0.032)$. However, there was no association between the CA125 concentration and tumor response for any line of chemotherapy (Fig. 2B).

Of the 30 patients that succumbed, $2(6.7 \%)$ had received second-line chemotherapy, 7 (23.3\%) third-line chemotherapy, 11 (36.7\%) fourth-line chemotherapy, and $10(33.3 \%)$ fifth-line chemotherapy as their last line of chemotherapy (Fig. 3A).

Whether the pre-treatment NLR, PLR, CA125 concentration and PS were associated with final-line chemotherapy was also examined in the present study. Pre-treatment NLR and PLR of patients with PS of 2 were significantly higher than those of patients with a PS of 0 prior to final 
A
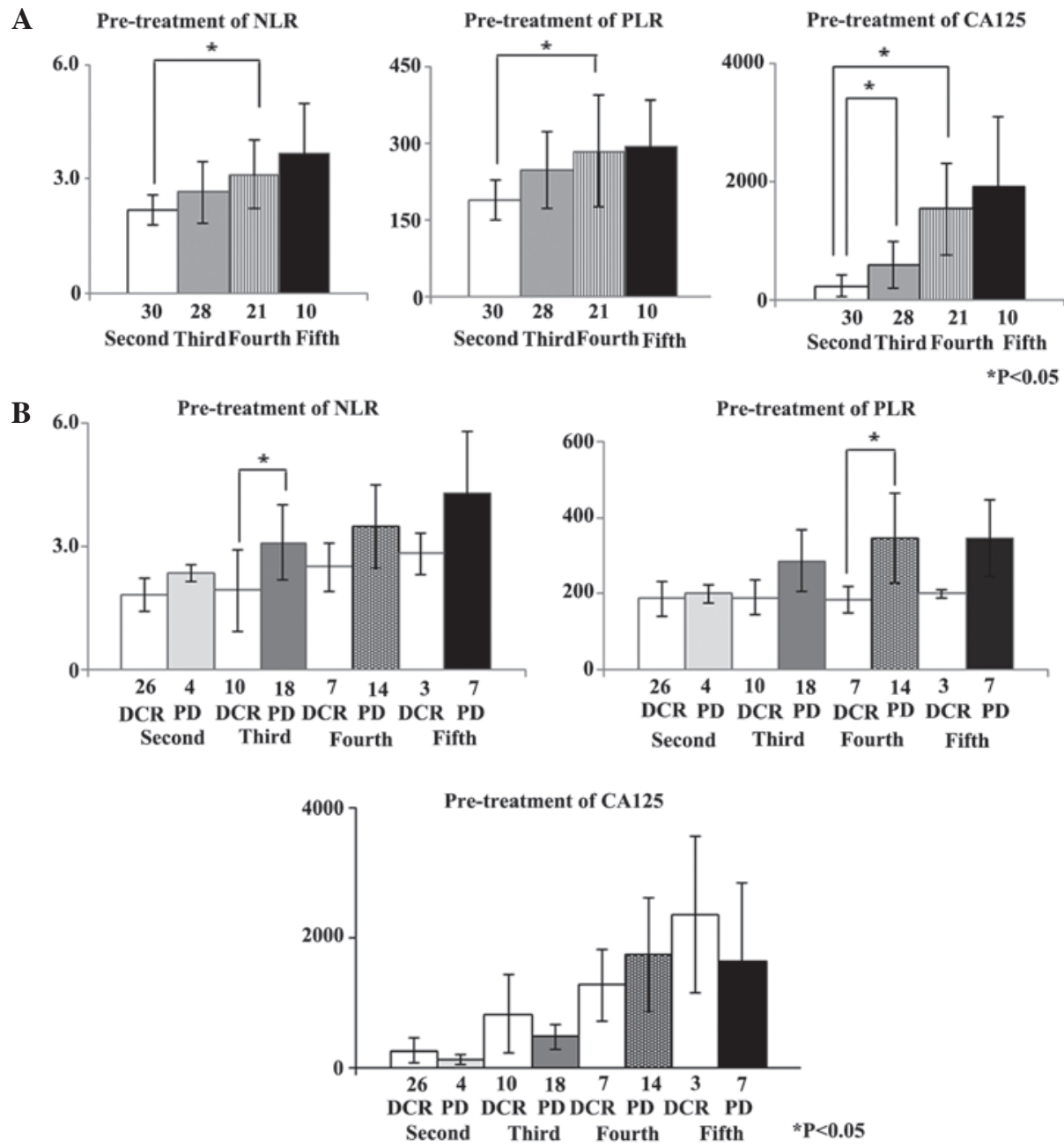

Figure 2. (A) Second to fifth-line chemotherapy: pre-treatment NLR, PLR and CA125 concentrations in patients with recurrent ovarian cancer. (B) Second- to fifth-line chemotherapy: tumor responses according to pre-treatment NLR, PLR, and CA125 concentrations in patients with recurrent ovarian cancer. NLR, neutrophil to lymphocyte ratio; PLR, platelet to lymphocyte ratio; CA125, cancer antigen 125; DCR, disease control rate (including complete and partial responses and stable disease); PD, progressive disease.
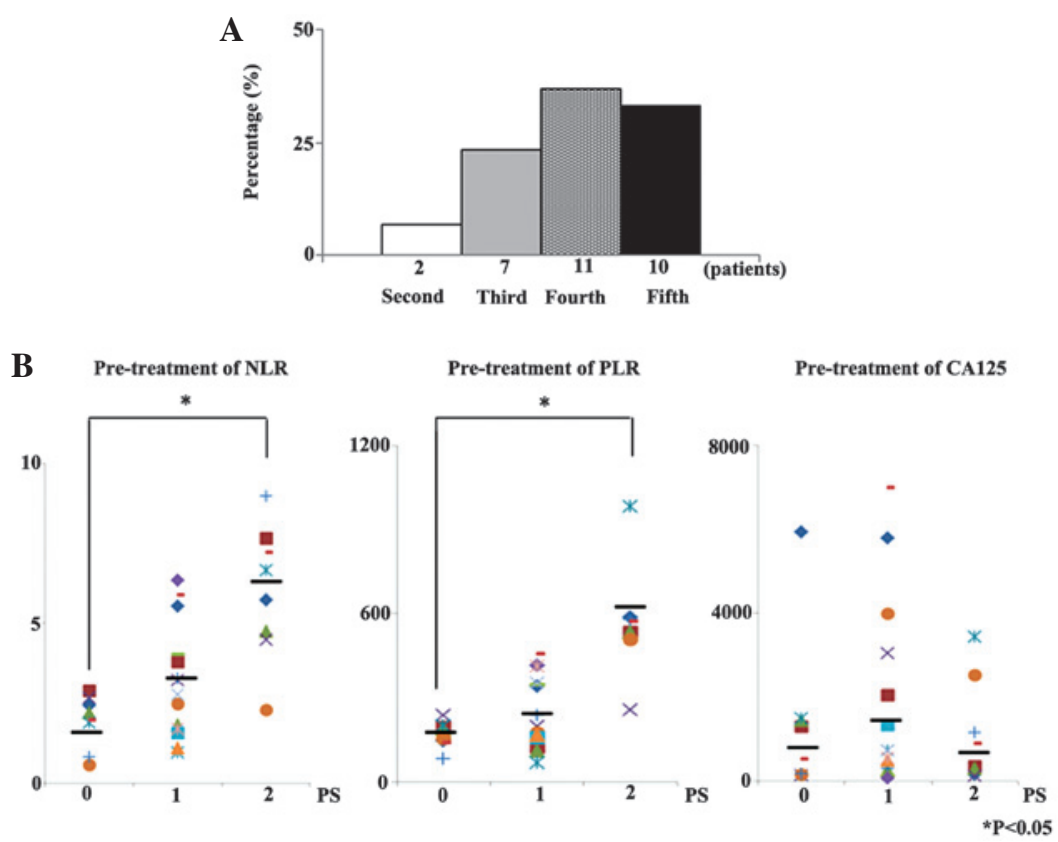

Figure 3. (A) Numbers of patients for whom second, third, fourth and fifth-line chemotherapy was the final chemotherapy. (B) Final-line chemotherapy pre-treatment NLR, PLR and CA125 concentrations according to PS. NLR, neutrophil to lymphocyte ratio; PLR, platelet to lymphocyte ratio; CA125, cancer antigen 125; PS, performance status. 
A

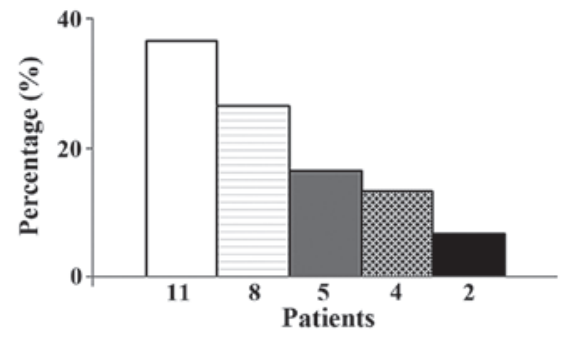

B

$\leq 100101-200201-300301-400401 \leq$ (Days)
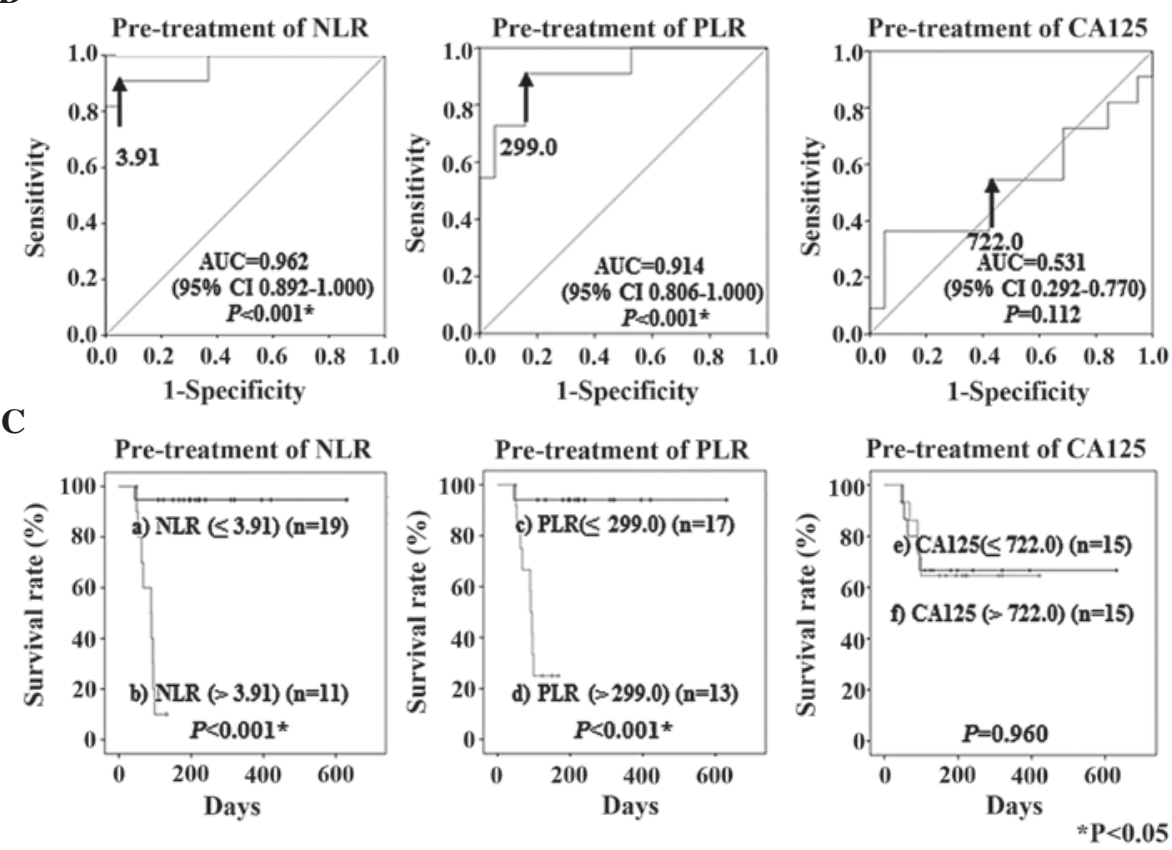

Figure 4. (A) Time between final-line chemotherapy and mortality in patients with recurrent ovarian cancer. (B) Receiver operating characteristic curves for the ability of pre-treatment NLR, PLR and CA125 concentrations to predict mortality within 100 days of final-line chemotherapy. The optimal cut-off value for pre-treatment NLR was 3.91 (AUC, 0.962; 95\% CI, 0.892-1.000; P<0.001), for PLR it was 299.0 (AUC, 0.914; 95\% CI, 0.806-1.000; P<0.001), and for CA125 concentrations it was $722.0 \mathrm{U} / \mathrm{ml}$ (AUC, 0.531; 95\% CI, 0.292-0.770; P=0.112). (C) Kaplan-Meier plots for the survival rates of 30 patients with recurrent ovarian cancer, based on the pre-treatment NLR, PLR and serum CA125 values. a), NLR $\leq 3.91$ ( $n=19)$; b), NLR >3.91 ( $n=11$ ); c), PLR $\leq 299.0$ ( $n=17$ ); d), PLR >299.0 ( $n=13)$, e) serum CA125 concentrations $\leq 722.0 \mathrm{U} / \mathrm{ml}(\mathrm{n}=15)$; and $\mathrm{f})$, serum CA125 concentrations $>722.0 \mathrm{U} / \mathrm{ml}$ ( $\mathrm{n}=15)$. NLR, neutrophil to lymphocyte ratio; PLR, platelet to lymphocyte ratio; CA125, cancer antigen 125; AUC, area under the curve; U/ml, units/ml.

line chemotherapy $(\mathrm{P}<0.001$ and $\mathrm{P}<0.001)$. However, CA125 concentration prior to final line chemotherapy was not associated with PS (Fig. 3B).

The time between final line chemotherapy and mortality was $\leq 100$ days in 11 patients (36.7\%), 101-200 days in 8 (26.7\%), 201-300 days in 5 (16.6\%), 301-400 days in 4 (13.3\%), and $\geq 401$ days in 2 (6.7\%) (Fig. 4A).

ROC curve analysis. ROC curve analyses were performed to determine the cut-off values for the pre-treatment NLR, PLR and CA125 concentrations that predicted mortality within 100 days of the failure of final line chemotherapy. These analyses identified a cut-off value for a pre-treatment NLR of 3.91 (AUC, 0.962; sensitivity, 90.9\%; specificity, 94.7\%), for a pre-treatment PLR of 299.0 (AUC, 0.914; sensitivity, 90.9\%; specificity, $84.2 \%$ ) and for a pre-treatment CA125 concentration of 722.0 (units $/ \mathrm{ml}$ ) (AUC, 0.531; sensitivity, 54.5\%; specificity, 52.6\%) (Fig. 4B). When patients were classified into the regions above and below each cut-off value for survival, Kaplan-Meyer curves of survival demonstrated that patients with a high NLR and PLR had significantly shorter survival times than patients with a low NLR and PLR $(\mathrm{P}<0.001$ and $\mathrm{P}<0.001$, respectively) (Fig. 4C).

Association between clinical factors and mortality. The association between clinical factors and mortality within 100 days of the failure of final-line chemotherapy was assessed by univariate and multivariate analyses. According to the univariate analyses, pre-treatment NLR $>3.91$, pre-treatment PLR $>299.0$ and PS 2 were significantly associated with mortality within 100 days of the failure of final-line chemotherapy ( $\mathrm{P}=0.001,0.005$ and 0.021 , respectively). Furthermore, multivariate analyses demonstrated that patients with high pre-treatment NLR had a significantly increased hazard ratio (14.128) for mortality within 100 days subsequent to failure of final line chemotherapy compared with patients without this risk factor $(\mathrm{P}=0.035)$ (Table II).

\section{Discussion}

Predictors of the outcome of multiple metastases in cancer patients with recurrence include factors associated with the 
response to chemotherapy (23). The decision to administer chemotherapy should consider various clinical factors, including PS, likely tumor response and survival. In particular, the prediction of the survival time is important. Evidence of the deterioration and adverse effects associated with chemotherapy should be monitored closely to avoid over-treatment (8).

In addition to the clinical predictors of the duration of survival, including the PS, factors associated with systemic inflammatory processes have been examined for possible roles as biomarkers of prognosis in various types of cancer (24). Neutrophils and T and B-lymphocytes have been suggested as prominent in inflammatory and immunological responses to tumors $(25,26)$. Inflammatory responses to tumors are mediated by the release from neutrophils of inflammatory cytokines, leukocytic and other phagocytic mediators that induce damage to cellular DNA, inhibit apoptosis and promote cancer-associated angiogenesis $(11,27,28)$. Platelets may be important for releasing potent mitogens or glycoproteins, such as platelet-derived growth factor, platelet factor 4 , transforming growth factor $\beta$, vascular endothelial growth factor and thrombospondin, which adhere to various cell types, including ovarian surface epithelium (29-33). Various ratios of neutrophil, platelet and lymphocyte counts, notably the NLR and PLR, have attracted the interest of investigators as possible markers of systemic inflammation, and therefore of prognosis (15-19). High pre-treatment NLR and PLR have reportedly been significantly associated with mortality in various types of cancer (12-16). High NLR is an independent indicator of poor prognosis in patients with epithelial ovarian cancer (34) and PLR is a better predictor of survival of these patients (35). However, no studies have reported correlations between the pre-treatment NLR and PLR, tumor response or pre-treatment CA125 concentrations and the survival of patients with recurrent ovarian cancer following chemotherapy. Therefore, in the present study, these associations were assessed.

The pre-treatment NLR, PLR and CA125 concentrations for patients that received second to fifth-line chemotherapy were calculated. The NLR and PLR prior to fourth-line chemotherapy were significantly greater than prior to second-line chemotherapy. Pre-treatment CA125 concentrations for patients that received third and fourth-line chemotherapy were significantly greater than for patients that received second-line chemotherapy. In addition, associations between pre-treatment NLR and PLR and DCR for each line of chemotherapy were assessed. Prior to third-line chemotherapy, patients that later developed PD had significantly greater pre-treatment NLR compared with those that achieved CR, PR and SD. Prior to fourth-line chemotherapy, patients that went on to develop PD had significantly greater PLR compared with patients that achieved CR, PR and SD.

The less sensitive a cancer is to chemotherapy, the more consideration treatment decisions require (19). The duration of survival is challenging to predict; however, poor PS is one of the strongest predictors of a short survival time $(8,24)$. In the present study, whether NLR, PLR and CA125 concentrations prior to final line chemotherapy were associated with PS was examined. PS was identified to be significantly correlated with the pre-treatment NLR and PLR. Furthermore, prior to final-line chemotherapy, patients with a worse PS (PS 2) had significantly greater NLR and PLR than patients with a better PS (PS 0).

In the present study, $36.7 \%$ of patients with recurrent epithelial ovarian cancer subsequent to chemotherapy received final line chemotherapy within 100 days prior to mortality. This is consistent with other studies, such as one study in which $30 \%$ of patients received final-line chemotherapy in the final month of life (36) and another in which $50 \%$ of patients received treatment in the final two months of life (37). One of the aims of the present study was to determine whether the NLR and PLR values and CA125 concentration prior to final-line chemotherapy may predict mortality within 100 days. ROC curve analyses were used to determine the optimal cut-off values for predicting mortality within 100 days of final line chemotherapy. The cut-off values for the pre-treatment NLR and PLR values and CA125 concentration were 3.91, 299.00 and 722 units $(\mathrm{U}) / \mathrm{ml}$, respectively, for survival for $<100$ days subsequent to final-line chemotherapy. According to univariate analyses using the determined cut-off values, pre-treatment NLR >3.91, PLR >299.0 and PS 2 were significantly associated with the outcomes of patients following failure of final-line chemotherapy. Multivariate analysis demonstrated that pre-treatment NLR (>3.91) was an independent negative indicator of the duration of survival time following the failure of final-line chemotherapy.

The limitations of the present study have been acknowledged. First, the number of patients was relatively small. Second, the duration of the follow-up was relatively short. Additional prospective studies with larger patient numbers and longer follow-up periods may provide more definitive data to clarify the significance of the findings.

In summary, the findings of the present study suggest that the pre-treatment NLR is an important predictor of the outcome of patients with recurrent ovarian cancer following chemotherapy.

\section{References}

1. Prat J; FIGO Committee on Gynecologic Oncology: Staging classification for cancer of the ovary, fallopian tube, and peritoneum. Int J Gynaecol Obstet 124: 1-5, 2014.

2. Cannistra SA: Cancer of the ovary. N Engl J Med 351: 2519-2529, 2004.

3. Clarke-Pearson DL: Clinical practice. Screening for ovarian cancer. N Engl J Med 361:170-177, 2009.

4. Covens A, Carey M, Bryson P, Verma S, Fung Kee, Fung M and Johnston M: Systematic review of first-line chemotherapy for newly diagnosed postoperative patients with stage II, III, or IV epithelial ovarian cancer. Gynecol Oncol 85: 71-80, 2002.

5. Parmar MK, Ledermann JA, Colombo N, du Bois A, Delaloye JF, Kristensen GB, Wheeler S, Swart AM, Qian W, Torri V, et al; ICON and AGO Collaborators: Paclitaxel plus platinum-based chemotherapy versus conventional platinum-based chemotherapy in women with relapsed ovarian cancer: The ICON4/AGO-OVAR-2.2 trial. Lancet 361: 2099-2106, 2003.

6. Alberts DS, Liu PY, Wilczynski SP, Clouser MC, Lopez AM, Michelin DP, Lanzotti VJ and Markman M; Southwest Oncology Group: Randomized trial of pegylated liposomal doxorubicin (PLD) plus carboplatin versus carboplatin in platinum-sensitive (PS) patients with recurrent epithelial ovarian or peritoneal carcinoma after failure of initial platinum-based chemotherapy (Southwest Oncology Group Protocol S0200). Gynecol Oncol 108: 90-94, 2008.

7. O'Brien ME, Yau T, Coward J, Hughes S, Papadopoulos P, Popat S, Norton A and Ashley S: Time and chemotherapy treatment trends in the treatment of elderly patients (age $>$ or $=70$ years) with non-small cell lung cancer. Clin Oncol (R Coll Radiol) 20: 142-147, 2008. 
8. Kim A, Fall P and Wang D: Palliative care: Optimizing quality of life. J Am Osteopath Assoc 105 (Suppl 5): S9-S14, 2005.

9. Renouf D, Kennecke H and Gill S: Trends in chemotherapy utilization for colorectal cancer. Clin Colorectal Cancer 7: 386-389, 2008.

10. Lee Y, Kim SH, Han JY, Kim HT, Yun T and Lee JS: Early neutrophil-to-lymphocyte ratio reduction as a surrogate marker of prognosis in never smokers with advanced lung adenocarcinoma receiving gefitinib or standard chemotherapy as first-line therapy. J Cancer Res Clin Oncol 138: 2009-2016, 2012.

11. Balkwill F and Mantovani A: Inflammation and cancer: Back to Virchow? Lancet 357: 539-545, 2001.

12. Coussens LM and Werb Z: Inflammation and cancer. Nature 420 860-867, 2002

13. Templeton AJ, McNamara MG, Šeruga B, Vera-Badillo FE, Aneja P, Ocaña A, Leibowitz-Amit R, Sonpavde G, Knox JJ, Tran $\mathrm{B}$, et al: Prognostic role of neutrophil-to-lymphocyte ratio in solid tumors: A systematic review and meta-analysis. J Natl Cancer Inst 106: dju124, 2014.

14. Templeton AJ, Ace O, McNamara MG, Al-Mubarak M, Vera-Badillo FE, Hermanns T, Seruga B, Ocaña A, Tannock IF and Amir E: Prognostic role of platelet to lymphocyte ratio in solid tumors: A systematic review and meta-analysis. Cancer Epidemiol Biomarkers Prev 23: 1204-1212, 2014.

15. Aliustaoglu M, Bilici A, Seker M, Dane F, Gocun M, Konya V, Ustaalioglu BB and Gumus M: The association of pre-treatment peripheral blood markers with survival in patients with pancreatic cancer. Hepatogastroenterology 57: 640-645, 2010.

16. Azab B, Bhatt VR, Phookan J, Murukutla S, Kohn N, Terjanian T and Widmann WD: Usefulness of the neutrophil-to-lymphocyte ratio in predicting short- and long-term mortality in breast cancer patients. Ann Surg Oncol 19: 217-224, 2012.

17. Kishi Y, Kopetz S, Chun YS, Palavecino M, Abdalla EK and Vauthey JN: Blood neutrophil-to-lymphocyte ratio predicts survival in patients with colorectal liver metastases treated with systemic chemotherapy. Ann Surg Oncol 16: 614-622, 2009.

18. Sarraf KM, Belcher E, Raevsky E, Nicholson AG, Goldstraw P and Lim E: Neutrophil/lymphocyte ratio and its association with survival after complete resection in non-small cell lung cancer. J Thorac Cardiovasc Surg 137: 425-428, 2009.

19. Yamanaka T, Matsumoto S, Teramukai S, Ishiwata R, Nagai Y and Fukushima M: The baseline ratio of neutrophils to lymphocytes is associated with patient prognosis in advanced gastric cancer. Oncology 73: 215-220, 2007.

20. Bast RC Jr, Klug TL, St John E, Jenison E, Niloff JM, Lazarus H, Berkowitz RS, Leavitt T, Griffiths CT, Parker L, et al: A radioimmunoassay using a monoclonal antibody to monitor the course of epithelial ovarian cancer. N Engl J Med 309: 883-887, 1983.

21. Oken MM, Creech RH, Tormey DC, Horton J, Davis TE, McFadden ET and Carbone PP: Toxicity and response criteria of the Eastern Cooperative Oncology Group. Am J Clin Oncol 5: 649-655, 1982.

22. Eisenhauer EA, Therasse P, Bogaerts J, Schwartz LH, Sargent D, Ford R, Dancey J, Arbuck S, Gwyther S, Mooney M, et al: New response evaluation criteria in solid tumours: Revised RECIST guideline (version 1.1). Eur J Cancer 45: 228-247, 2009.
23. Prommer E: Guidelines for the use of palliative chemotherapy AAHPM Bulletin 5: 2-13, 2004.

24. McIllmurray M: Symptom management. In: Oxford Textbook of Palliative Medicine. Doyle D, Hanks G, Cherny NI and Calman K (eds). 3rd edition. Oxford University Press, New York, NY, 229-239, 2004.

25. Lin X, Li W, Lai J, Okazaki M, Sugimoto S, Yamamoto S, Wang X, Gelman AE, Kreisel D and Krupnick AS: Five-year update on the mouse model of orthotopic lung transplantation: Scientific uses, tricks of the trade, and tips for success. J Thorac Dis 4: 247-258, 2012.

26. Schreiber RD, Old LJ and Smyth MJ: Cancer immunoediting: Integrating immunity's roles in cancer suppression and promotion. Science 331: 1565-1570, 2011.

27. Jackson JR, Seed MP, Kircher CH, Willoughby DA and Winkler JD: The codependence of angiogenesis and chronic inflammation. FASEB J 11; 457-465, 1997.

28. McMillan DC, Canna K and McArdle CS: Systemic inflammatory response predicts survival following curative resection of colorectal cancer. Br J Surg 90: 215-219, 2003.

29. Assoian RK and Sporn MB: Type beta transforming growth factor in human platetlets: Release during platelet degranulation and action on vascular smooth muscle cells. J Cell Biol 102: 1217-1223, 1986

30. Dubernard V, Arbeille BB, Lemesle MB and Legrand C: Evidence for an alpha-granular pool the cytoskeletal protein alpha-actinin in human platelets that redistributes with the adhesive glycoprotein thrombospondin-1 during the exocytotic process. Arterioscler Thromb Vasc Biol 17: 2293-2305, 1997.

31. Kaplan KL, Broekman MJ, Chernoff A, Lesznik GR and Drillings M: Platelet alpha-granule proteins: Studies on release and subcellular localization. Blood 53: 604-618, 1979.

32. Qian X and Tuszynski GP: Expression of thrombospondin-1 in cancer: A role in tumor progression. Proc Soc Exp Biol Med 212: 199-207, 1996

33. Dabrow MB, Francesco MR, McBrearty FX and Caradonna S: The effects of platelet-derived growth factor and receptor on normal and neoplastic human ovarian surface epithelium. Gynecol Oncol 71: 29-37, 1998

34. Cho H, Hur HW, Kim SW, Kim SH, Kim JH, Kim YT and Lee K: Pre-treatment neutrophil to lymphocyte ratio is elevated in epithelial ovarian cancer and predicts survival after treatment. Cancer Immunol Immunother 58: 15-23, 2009.

35. Asher V, Lee J, Innamaa A and Bali A: Preoperative platelet lymphocyte ratio as an independent prognostic marker in ovarian cancer. Clin Transl Oncol 13: 499-503, 2011.

36. Matsuyama R, Reddy S and Smith TJ: Why do patients choose chemotherapy near the end of life? A review of the prospective of those facing death from cancer. J Clin Oncol 24: 3490-3496, 2006.

37. Keam B, Oh DY, Lee SH, Kim DW, Kim MR, Im SA, Kim TY, Bang YJ and Heo DS: Aggressiveness of cancer-care near the end-of-life in Korea. Jpn J Clin Oncol 38: 381-386, 2008. 